\title{
The 8th Annual State of SoSyM Report
}

\author{
Editorial for the SoSyM Issue 2009/01
}

Published online: 29 November 2009

(C) Springer-Verlag 2009

Another year has gone by and we are happy to report that the International Journal on Software and Systems Modeling (SoSyM) is doing well. As we have done on previous anniversaries, we take this opportunity to give a "state of the journal" report and to acknowledge the reviewers, editors, and publication staff who have contributed to the journal's very good performance in the past year.

\section{The 2009 "State of the Journal" Report}

We continue to have a steady stream of submissions and the number of new submissions rose in 2009, as it has since the journal's inception. In 2009, 465 new manuscripts were submitted to SoSyM. Of these, 381 were reviewed for regular issues, and 84 were reviewed for special sections. The average number of days from submission to a final decision (accept or reject) was approximately 180 in 2009 . The average number of days from first submission to a first decision was approximately 116 . The number of months to online publication of an accepted paper has continued to drop and is now just under 2 months.

The current acceptance rate for regular papers is $20.4 \%$; $1.9 \%$ of the accepted papers were accepted without revision, $11.2 \%$ were accepted after major revision, and $7.3 \%$ were accepted after minor revision. The acceptance rate for special section papers is $35.1 \%$, of which $19.5 \%$ of the accepted papers were accepted after major revision and $15.6 \%$ were accepted after minor revision. The current publication pipeline is approximately 1 year, that is, we have approximately 1 year's worth of papers to be published.

The timely turnaround of papers and the high quality of accepted papers are a result of the excellent effort the editors and reviewers put into the review process. We understand how difficult it is to balance editorial and review responsibilities with other pressing responsibilities and thus we greatly appreciate their effort.

In 2009, Professor August-Wilhelm Scheer resigned from the editorial board. Professor Scheer was on the board, since the inception of the journal and he could always be relied upon to complete his review assignments in a professional and timely manner. We take this opportunity to thank Professor Scheer for his invaluable service to the journal.

Recently, we added three highly regarded researchers in the software and system modeling area to the editorial board. It gives us great pleasure to welcome the following new editorial board members:

- Marsha Chechik, Department of Computer Science, University of Toronto, Canada

- Richard Paige, Department of Computer Science, University of York, UK

- Antonio Valecillo, Dept. Lenguajes y Ciencias de la Comptacion, Universidad de Malaga, Spain

We look forward to working with our extended editorial board in 2010 .

The journal's growing reputation is a direct result of the effort and expertise of the editors and reviewers. Below, we list the reviewers who reviewed one or more papers for the journal in the last year. A complete list of reviewers can be found on our website http://www.sosym.org/.

Bernhard Aichernig, Marco Aiello, Neta AizenbudReshef, Bastien Amar, Kelly Androutsopoulos, Sven Apel, Hazeline Asuncion, Colin Atkinson, Emine Aydal, Robert Baillargeon, Paul Baker, Olivier Barais, Luciano Baresi, Don Batory, Benoit Baudry, Bernhard Bauer, Bernhard Beckert, Nelly Bencomo, Stefan Berghofer, Simona Bernardi, Mireille Blay-Fornarino, Olivier Boissier, Behzad Bordbar, 
Artur Boronat, Thouraya Bouabana-Tebibel, Achim D. Brucker, Jordi Cabot, Jaelson Castro, Walter Cazzola, María Victoria Cengarle, Antonio Cerone, Antonio Cicchetti, Tony Clark, Manuel Clavel, Sholom Cohen, Benoit Combemale, Steve Cook, Alexandre Luis Correa, Vittorio Cortellessa, Andrea D'Ambrogio, Zhe Dang, Hung Dang Van, Eric Dashofy, Guglielmo De Angelis, Miguel A. de Miguel, Jerome Delatour, Joerg Desel, Davide Di Ruscio, Ada Diaconescu, Joerg Doerr, Thorsten Dollmann, Fernando Luís Dotti, Emmanuel Dubois, Marina Egea Gonzalez, Alexander Egyed, Karsten Ehrig, George Eleftherakis, Angelina Espinoza, Huascar Espinoza, Antonio Estevez, Jean-Remy Falleri, Eduardo Buglioni Fernandez, Jorge Fox, Giuliana A. Franceschinis, Piero Fraternali, Felix Garcia, Mareclla Genero, Geri Georg, John Georgas, Jeremy Gibbons, Holger Giese, Rosario Girardi, Stefania Gnesi, Aniruddha Gokhale, Paul Grace, Emanuel S. Grant, Vincenzo Grassi, Jeff Gray, Andreas Griesmayer, Hans-Gerhard Gross, Paul Gruenbacher, Ibrahim Habli, Axel Hahn, Brahim Hamid, Moritz Hammer, Jens Happe, Klaus Havelund, Kelly Hayhurst, Mats Heimdahl, Constance L. Heitmeyer, Mike Hinchey, Robert Hirschfeld, Andreas Hoffmann, Chris Holmes, John Gordon Hosking, Michaela Huhn, Hardi Hungar, Maria-Eugenia Iacob, Frank InnerhoferOberperfler, Muhammad Zohaib Zafar Iqbal, Bengt Jonsson, Bernhard Josko, Jan Juerjens, Günter Karjoth, Gabor Karsai, Pierre Kelsen, Garrin Kimmell, Alexander Knapp, Guenter Kniesel, Nora Koch, Samuel Kounev, Máté Kovács, Padmanabhan Krishnan, Vinay Kulkarni, Ludwik Kuzniarz, Marcel Kyas, Philippe Lahire, Jaejoon Lee, Katrina Leyking, Torsten Lodderstedt, Mass Soldal Lund, Radu Marinescu, Grant Martin, Martínez Rebollar Martínez Rebollar, Dan Matheson, Ilaria Matteucci, Raimundas Matulevicius, Peter Mehlitz, Tom Mens, Stephan Merz, Dragan Milicev, Mark Minas, Angelo Morzenti, Haralambos Mouratidis, Sadaf Mustafiz, Clementine Nebut, Thomas Noll, Jon Oldevik, Gøran K. Olsen, Claus Pahl, Richard Paige, Marc Pantel, Ioannis Parissis, Roberto Passerone, Susanne Patig, Vicente Pelechano, Greg Peterson, Dorina C. Petriu, Alfonso Pierantonio, Lee Pike, Simon Poulding, Alexander Pretschner, Oliver Radfelder, Wolfgang Reisig, Arend Rensink, Kai Richter, Mark Richters, Steve Riddle, Sabine Rittmann, José Rivera, Noemi Rodriguez, Markus Roggenbach, Raul Rojas, Philippe Roose, Judith Rosseboe, Ella Roubtsova, Nicolas Rouquettes, Ragnhild Kobro Runde, Eric Rutten, Antonino Sabetta, Pete Sawyer, Martin Schindler, Bernd Schmidt, Pierre-Yves Schobbens, Johann Schumann, Andy Schürr, Stefan Schwoon, Christian Seel, Mirko Seifert, Viviane Torres Silva, Devon Simmonds, Dennis B. Smith, Colin F. Snook, Arnor Solberg, Bjornar Solhaug, Jonathan Sprinkle, Erlend Stav, Martin Steffen, Dominik Stein, Perdita Stevens, Jing Sun, Daniel Sundmark, Jun Suzuki, Kuldar Taveter, Bedir Tekinerdogan, Ernest Teniente, Luca Tesei, Paolo
Tonella, Yasemin Topaloglu, Mario Trapp, Stavros Tripakis, Dan Turk, Antonio Vallecillo, Andre van der Poll, M. Birna van Riemsdijk, Bert Vanhooff, Sander Vermolen, Steven Voelkel, Dennis Wagelaar, Stale Walderhaug, Bruce Watson, Jules White, Reinhard Wilhelm, Will Winsborough, Andreas Winter, Sebastian Winter, Peter Wong, Dianxiang Xu, Emmanuele Zambon, Damiano Zanardini, and Jing Zhang

We also take this opportunity to thank the Springer publishing staff, namely: Hermann Engesser, Gabriele Stjepanovic, and Dorothea Glaunsinger. They all provided excellent assistance during the year. In particular, Hermann Engesser continuously provided us with indispensable assistance on all matters related to the management of the journal.

Last, but not the least, we thank our assistant editors, namely Geri Georg who handles regular papers, and Martin Schindler who handles special and theme sections, expert papers, prepares all the statistical data, including the data presented in this editorial, and manages the publication process for all accepted papers.

The journal's reputation and quality is a direct result of the outstanding support provided by authors, reviewers, editors, and the publishing staff. We, the editors-in-chief, are committed to ensuring that the journal continues to play an important role in the dissemination of knowledge in the software-based system modeling community. Finally, we thank, the reader for supporting SoSyM. Please do not hesitate to contact us with your comments about the journal.

\section{Contents in this issue}

This issue includes selected papers from MODELS 2007. These papers are introduced in an editorial by Gregor Engels, who served as Program Chair for MODELS'07. There are also two regular papers in this issue.

The first regular paper, "Process Mining: A Two-Step Approach to Balance Between Underfitting and Overfitting" by Wil M. P. Aalst, Vladimir Rubin, Eric Verbeek, Boudewijn Dongen, Ekkart Kindler, and Christian Günther, deals with a two-step process of creating process models in form of petri nets, using event logs. The authors address the problem of overfitting and underfitting during process mining, and propose a solution that they formally describe. An implementation utilizing ProM is described.

In the second regular paper, "Incorporating Message Weights in UML-based Analysis of Behavioral Dependencies in Distributed Systems", the author Vahid Garousi defines a number of metrics for behavioral dependency in distributed software systems. Behavioral dependency indicates the extent to which the functionality of one system entity depends on another, and is relevant for a variety of purposes, such as performing risk analysis or load planning. The author 
defines dependency metrics based on the weighted message exchanges and gives us an example to illustrate their potential usefulness.

We hope you enjoy reading the articles in this issue. We also hope you enjoyed reading all the papers that were published in SoSyM this year.
Robert France, Bernhard Rumpe Editors in Chief

Geri Georg, Martin Schindler Assistant Editors 